\title{
2nd Annual National ABCD IPN-UK meeting
}

\author{
Dr Suma Sugunendran ${ }^{1}$ and Dr Peter Jennings ${ }^{2}$ report from the 2 nd \\ Annual National ABCD Insulin Pump Network UK meeting in Belfast, \\ Ireland, on 10th May 2017
}

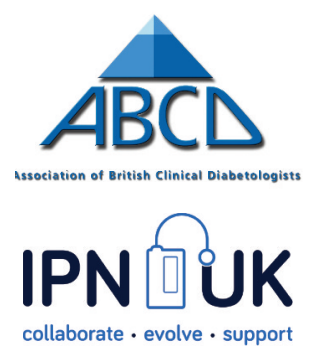

\begin{abstract}
Introduction
The 2nd annual national ABCD Insulin Pump Network UK (IPN-UK) meeting took place in the magnificent iconic venue of Titanic Belfast. With the famous Titanic ballroom staircase in the background to set the scene, Dr Emma Wilmot, Chair of IPN-UK, opened the meeting with an overview of the success of the IPN-UK over the previous year. This was followed by an update from the centre from Dr Partha Kar, National Associate Clinical Director for diabetes at NHS England. Quality improvement in type 1 diabetes is one of his key priorities, and he challenged the audience to suggest some key metrics which could be used to measure the quality of insulin pump services in the future.
\end{abstract}

\section{Papers presented at the meeting}

A key recurring topic mentioned by $\mathrm{Dr}$ Kar and also Dr Peter Hammond and Dr Nick Oliver was the REPOSE trial. ${ }^{1}$ As readers will be aware, the REPOSE trial was recently published in the BMJ. The main aim of the study was to answer the questions 'Are pumps for all?'. The short answer is no. A group of patients who did not meet the TA151 guidance for insulin pump therapy were randomised to dose adjusted for normal eating (DAFNE) and multiple daily injections (MDI) or DAFNE and an insulin pump. Overall there was an improvement in $\mathrm{HbA}_{1 \mathrm{c}}$ and severe hypoglycaemia in both arms, but insulin pump therapy did not result in a significant reduction in $\mathrm{HbA}_{1 c}$ compared with the MDI arm. The general consensus among the experts regarding the REPOSE study was that it should be taken in context, given that the study population would not have been eligible for insulin pump therapy as per NICE TA151 guidance. The study has highlighted the importance of structured education, its role in increasing the effectiveness of self-management and that MDI therapy is a perfectly reasonable option for glycaemic control. Although blanket adoption of insulin pump therapy is not advocated, its role is still important in subgroups of patients - for example, those meeting the NICE criteria. Unfortunately, as discussed by the IPN committee in the same issue of this journal, the REPOSE study has been misinterpreted by some. Dr Kar reassured healthcare professionals and patient groups about the implications of the REPOSE study. Insulin pump therapy access will continue as per the NICE TA151 guidelines and the IPN-UK committee will release a statement to this effect.

Dr Bob Young, Lead for the National Insulin Pump Audit, detailed the 2016 National Insulin Pump Audit (NIPA) results such as the varied use of continuous subcutaneous insulin infusion (CSII) across centres from $5 \%$ to $>50 \%$ of patients with type 1 diabetes. He also highlighted the fact that type 1 diabetes pump users were more likely to achieve their treatment targets and achieve a lower $\mathrm{HbA}_{1 \mathrm{c}}$ than those not using CSII. Delegates discussed possibilities for improved reporting and recording of hypoglycaemia and standardising clinic templates to capture relevant data. Dr Young highlighted the fact that an increased number of participating centres resulted in improved data quality from 2015 NIPA. The audit team is also keen to improve data quality and participation in subsequent years. It is anticipated that the 2018 audit will include a service level audit for both insulin pump therapy and type 1 diabetes care in general.

Peter Hammond updated delegates on the latest developments in diabetes technologies, with insight into the new insulin pumps including the Kaelido insulin pump, Medtronic 670G and Medtrum semi-closed loop. He mentioned that the evidence for insulin pump use in type 2 diabetes was developing and presented the OpT2mise study extension.

Vey relevant to our clinical practice, there are some interesting studies on infusion set changes. Infusion sets should be changed every 3 days to minimise the risk of occlusion and/or hyperglycaemia, except in pregnancy when it is advised that sets are changed every 2 days. $^{2}$

Professor Nick Oliver from Imperial College London then explored evidence relating to an interesting question becoming very relevant to current practice: 'Which technology should come first when intensifying type 1 diabetes management: CSII or continuous glucose monitoring (CGM)?' CGM has a positive impact on $\mathrm{HbA}_{1 c}$, time in range and hypoglycaemia reduction, regardless of insulin delivery modality. However, the persistence of $\mathrm{HbA}_{1 c}$ drop with CGM is dose-dependent (i.e. how continuously the sensors are used). Although the use of both pumps and CGM has a positive influence on avoiding hypoglycaemia, CGM is likely to be the better option for people with hypoglycaemic unawareness who rely on alarms to alert them to impending hypoglycaemia.

\section{Case studies}

Three clinical conundrums submitted by delegates underwent detailed analysis by an IPN-UK expert panel (Dr Pratik Choudhary, Dr Peter Hammond and Mrs Geraldine Gallen) with lively discussions among the delegates. Numerous suggestions for optimising CSII and CGM were raised. It was stressed that it is important that we use downloads routinely in the clinic settings and know how to interpret them. Cases were reviewed and a number of observations made. Hyperglycaemia can often be a late response following hypoglycaemia correction and many of the hypoglycaemias occur after a correction dose if the insulin sensitivity factor is too aggressive. Mastering the skills of interpreting CSII and CGM downloads and adjusting settings are essential to providing quality diabetes care. The importance of screening for diabetic distress was also highlighted.

\section{Workshops}

There were three interactive workshops which gave excellent practical tips on problematic hypoglycaemia, exercise and use of the Freestyle Libre.

Dr Pratik Choudhary, leading the hypoglycaemia workshop, made a very interesting and thought-provoking observation. Although we do the screening for nephropathy with an incidence of $15-40 \%$ and even thyroid with an even lesser incidence, we do not routinely screen for significant hypoglycaemia and hypoglycaemic awareness, which 
has a prevalence of more than $50 \%$ in patients with longstanding diabetes. ${ }^{3}$ It is very important we use validated questionnaires like the Gold score to detect and quantify hypoglycaemic awareness. ${ }^{4,5}$ For persistent hypoglycaemia, in addition to common causes like renal failure, gastroparesis and adrenal insufficiency, look for unpredictable insulin action (like lipodystrophy and insulin antibodies), other endocrine abnormalities such as growth hormone deficiency and mismatch of insulin need and requirement.

The exercise workshop led by Dr Alistair Lamb gave an in-depth understanding of exercise physiology and practices in type 1 diabetes which included the following points:

- Fear of hypoglycaemia is the most important factor preventing people from embracing a more active lifestyle.

- If the pre-exercise glucose is higher, it is ideal to start with aerobic exercise which will reduce glucose followed by anaerobic exercise which tends to raise glucose. If the pre exercise glucose is on the lower side, it would be a good idea to start with anaerobic exercise which will tend to increase the glucose.

- After prolonged severe hypoglycaemia, avoid exercise and take more carbohydrates.

A highlight of the afternoon was an insightful session from international guest speaker and founder of 'Sugar Surfing', Dr Steven Ponder, a paediatrician from Texas who was diagnosed with type 1 diabetes at a young age. $6 \mathrm{He}$ is driven by a passion to revolutionise glycaemic management and give all the necessary tools to patients and their families to allow them to make appropriate decisions. He gave a fascinating insight into the decision skills and matrix for 'microbolusing' and 'microcarbing' both reactively and proactively to achieve very little glycaemic variability in order to obtain a tight $\mathrm{HbA}_{1 c}$ (even less than 6\%) without hypoglycaemia. He describes his tool as 'cranial pancreas'. It needs a lot of dedication and commitment. It might not be every patient's cup of tea, but could be an option for the most dedicated few. He is offering to give the electronic version free for newly diagnosed patients with type 1 diabetes (www.sugarsurfing.com or the book 'Sugar surfing: How to manage type 1 diabetes in a modern world') to all members of the IPN-UK.

In addition to the technical aspects of diabetes self-care, he gave us an insight into the psychology of success and some very pertinent tips. The importance of avoiding use of the terminologies 'good' and 'bad' with glucose levels has huge significance. It is important not to judge and to use alternative terminologies like high/low/in range instead. It is so crucial that we as healthcare professionals reward the effort and not the results and give patients a goal for normal life. $\mathrm{HbA}_{1 \mathrm{c}}$ and glucose are only a means to an end. This dialogue and non-judgemental approach should be the norm for routine care of patients with type 1 patients from the point of diagnosis.

The whole day was thoroughly enjoyable and superbly paced, and it was not until the end of the meeting that we were fully able to appreciate the grandeur of the venue. Titanic Belfast is certainly worth a visit if you have a weekend to spare. We look forward to another exciting and informative meeting next year.

\section{References}

1. The REPOSE Study Group. Relative effectiveness of insulin pump treatment over multiple daily injections and structured education dur- ing flexible intensive insulin treatment for type 1 diabetes: cluster randomised trial (REPOSE). BMJ 2017;356:j1285.

http:dx.doi.org/10.1136/bmj.j1285.

2. Pfützner $A$, Sachsenheimer D, Grenningloh $M$, et al. Using insulin infusion sets in CSII for longer than the recommended usage time leads to a high risk for adverse events: results from a prospective randomized crossover study. J Diabetes Sci Technol 2015;9:1292-8. http:dx.doi.org/10.1177/1932296815604438

3. UK Hypoglycaemia Study Group. Risk of hypoglycaemia in types 1 and 2 diabetes: effects of treatment modalities and their duration. Diabetologia 2007;50:1140-7.

4. National Institute for Health and Care Excellence (NICE). Type 1 diabetes in adults: diagnosis and management (2015 updated 2016). NICE guideline NG17.

https://www. nice.org.uk/guidance/ng17

5. Geddes J, Wright RJ, Zammitt N, Deary IJ, Frier BM. An evaluation of methods of assessing impaired awareness of hypoglycaemia in type 1 diabetes. Diabetes Care 2007;30:1868-70.

6. Sugar Surfing ${ }^{\mathrm{TM}}$. www.sugarsurfing.com

${ }^{1}$ Consultant, Derby Teaching Hospitals NHS Foundation Trust, Royal Derby Hospital, Derby, UK 2Senior Teaching Fellow, University of Warwick and Diabetes Specialist Nurse, Royal Derby Hospital, Derby, UK

Correspondence: Dr Suma Sugunendran Derby Teaching Hospitals NHS Foundation Trust, Royal Derby Hospital, Uttoxeter Road, Derby DE22 3NE, UK

Email: suma.sugunendran@nhs.net

http://dx.doi.org/10.15277/bjd.2017.128 Br J Diabetes 2017;17:79-80 